\title{
Characterization of inflammatory bowel disease in hospitalized elderly patients in a large central Canadian health region
}

\author{
Peter Stepaniuk BSc(Hons) ${ }^{1}$, Charles N Bernstein $\mathrm{MD}^{1,2}$, Zoann Nugent PhD ${ }^{1,2,3}$, Harminder Singh MD MPH ${ }^{1,2,4}$
}

\begin{abstract}
P Stepaniuk, CN Bernstein, Z Nugent, H Singh. Characterization of inflammatory bowel disease in hospitalized elderly patients in a large central Canadian health region. Can J Gastroenterol Hepatol 2015;29(5):274-278.
\end{abstract}

OBJECTIVE: To determine differences in phenotype and treatment among hospitalized elderly and young patients with inflammatory bowel disease (IBD), and the utility of International Classification of Diseases, 10th Revision (ICD)-10 codes in hospital discharge abstracts in diagnosing IBD in elderly patients.

METHODS: A large Canadian health region hospitalization discharge database was used to identify elderly ( $>65$ years of age) and young ( 19 to 50 years of age) patients with IBD admitted between April 1, 2007 and March 31, 2012, and a random sample of elderly patients with other colonic conditions. Medical records were reviewed to confirm IBD diagnosis and extract clinical information. The characteristics of elderly versus young hospitalized IBD patients and accuracy of ICD-10 IBD discharge codes in the elderly were assessed.

RESULTS: One hundred forty-three elderly and 82 young patients with an IBD discharge diagnosis, and 135 elderly patients with other gastrointestinal discharge diagnoses were included. Elderly IBD patients were less likely to have ileocolonic Crohn disease (21.4\% versus $50.9 \% ; \mathrm{P}=0.001)$, more likely to be prescribed 5 -aminosalicylates $(61 \%$ versus $43 \% ; \mathrm{P}=0.04)$, and less likely to be prescribed biologics $(6 \%$ versus $21 \% ; \mathrm{P}=0.016)$ or immunomodulators ( $21 \%$ versus $42 \%$; $\mathrm{P}=0.01$ ). The sensitivity, specificity and positive predictive value of a single ICD code for CD were $98 \%, 96 \%$ and $94 \%$, respectively, and for ulcerative colitis (UC) were $98 \%, 92 \%$ and $70 \%$, respectively.

CONCLUSIONS: Treatment approaches in elderly patients were different than in younger IBD patients despite having disease sufficiently severe to require hospitalization. While less accurate in UC, a single ICD-10 IBD code was sufficient to identify elderly CD and UC hospitalized patients.

Key Words: Crohn disease; Elderly; Hospitalized patient outcomes; Ulcerative colitis

Tnflammatory bowel disease (IBD), including Crohn disease (CD) and ulcerative colitis (UC), are increasing in prevalence in both developed and developing countries around the world. Because IBD has minimal impact on mortality and, as populations are aging, the numbers of elderly individuals living with IBD are increasing (1). In addition, several studies have reported increasing incidence of IBD in the elderly (1-3). Hence, it is important to improve our knowledge base regarding the epidemiology and management of elderly individuals with IBD.

There may be differences in how the elderly and younger patients with IBD manifest with their disease, and how they are managed (4). Furthermore, elderly patients may present with symptoms that can resemble other common gastrointestinal conditions common to the

\section{La caractérisation des maladies inflammatoires de l'intestin chez les patients âgés hospitalisés dans une grande région sociosanitaire du centre du Canada}

\begin{abstract}
OBJECTIF : Déterminer les différences de phénotype et de traitement entre les personnes âgées et les patients plus jeunes hospitalisés à cause d'une maladie inflammatoire de l'intestin (MII) ainsi que l'utilité des dix codes de la Classification internationale des maladies, $10^{e}$ révision (CIM-10) dans les résumés de congés hospitaliers pour diagnostiquer les MII chez les patients âgés.
\end{abstract}

MÉTHODOLOGIE : Les chercheurs ont puisé dans une vaste base de données des congés hospitaliers d'une grande région sociosanitaire canadienne pour répertorier les patients âgés (plus de 65 ans) et plus jeunes (de 19 à 50 ans) atteints d'une MII qui avaient été hospitalisés entre le $1^{\mathrm{er}}$ avril 2007 et le 31 mars 2012, de même qu'un échantillon aléatoire de patients âgés atteints d'autres affections du colon. Ils ont examiné les dossiers médicaux pour confirmer le diagnostic de MII et extraire l'information clinique. Ils ont évalué les caractéristiques des personnes âgées hospitalisées à cause d'une MII par rapport à celles des plus jeunes et la précision des codes de congé de la CIM-10 utilisés dans leur dossier.

RÉSULTATS : Ont été inclus dans l'étude 145 patients âgés et 82 patients plus jeunes ayant un diagnostic de MII à leur congé, ainsi que 135 patients âgés ayant un autre diagnostic de maladie intestinale à leur congé. Les patients âgés atteints d'une MII étaient moins susceptibles d'avoir une maladie de Crohn $(\mathrm{MC})$ iléocolique (21,4 \% par rapport à 50,9\%; $\mathrm{P}=0,001)$, plus susceptibles de se faire prescrire des 5 -aminosalicylates (61 \% par rapport à $43 \% ; \mathrm{P}=0,04)$ et moins susceptibles de se faire prescrire des produits biologiques ( $6 \%$ par rapport à $21 \% ; \mathrm{P}=0,016)$ ou des immunomodulateurs ( $21 \%$ par rapport à $42 \% ; \mathrm{P}=0,01$ ). La sensibilité, la spécificité et la valeur prédictive positive d'un seul code de CIM pour la MC s'élevaient à $98 \%$, $96 \%$ et $94 \%$, respectivement, et, pour la colite ulcéreuse (CU), à $98 \%, 92 \%$ et $70 \%$, respectivement.

CONCLUSIONS : La conduite thérapeutique chez les patients âgés était différente de celle adoptée chez les patients plus jeunes atteints d'une MII, même si la maladie était assez grave pour exiger une hospitalisation. Bien qu'il soit moins précis en cas de CU, un seul code de CIM-10 suffisait pour distinguer les patients âgés hospitalisés à cause d'une MC et d'une CU.

elderly such as diverticulitis, ischemic colitis and infective colitis. More information is required to discern just how elderly and younger patients differ in terms of disease phenotype and treatment, particularly among hospitalized patients. There are limited comparative data regarding the clinical characteristics and current management in routine clinical practices between hospitalized elderly and young patients with confirmed IBD.

International Classification of Diseases (ICD) codes, the primary diagnostic coding system used in hospital settings, are used to identify individuals with chronic diseases and to generate epidemiological data. Very few studies have examined the accuracy of using these diagnostic codes to identify the elderly IBD population, which is important to assess specifically because of the other common gastrointestinal

\footnotetext{
${ }^{1}$ Internal Medicine, University of Manitoba; ${ }^{2}$ University of Manitoba IBD Clinical and Research Centre; ${ }^{3}$ CancerCare Manitoba, Department of

Epidemiology and Cancer Registry; ${ }^{4}$ Community Health Sciences, University of Manitoba, Winnipeg, Manitoba

Correspondence: Dr Harminder Singh, Section of Gastroenterology, University of Manitoba, 805-715 McDermot Avenue, Winnipeg, Manitoba R3E 3P4.

Telephone 204-480-1311, fax 204-789-3972, e-mail harminder.singh@umanitoba.ca
}

Received for publication January 18, 2015. Accepted January 25, 2015 
conditions in the elderly. Some studies exploring the epidemiology of IBD in elderly populations are undertaken using health care administrative databases; hence, it is important to determine how robust administrative data definitions are in these populations.

The objectives of the current study were to compare the clinical course, presentation and management of elderly and younger individuals hospitalized with IBD, and to determine the accuracy of hospital discharge diagnosis codes for IBD in the elderly.

\section{METHODS}

The present study was approved by the University of Manitoba Health Research Ethics Board (Winnipeg, Manitoba). The present study was performed in Winnipeg (Manitoba), an ethnically diverse city located in the centre of Canada with a metropolitan area containing approximately 780,000 individuals. Manitoba Health is the government agency funding universal health care in the province. The Winnipeg Regional Health Authority (WRHA) administers all six hospitals in the city of Winnipeg. Two of the hospitals (Health Sciences Centre and St Boniface Hospital) are tertiary academic hospitals situated close to the city core, while the four other hospitals (Grace Hospital, Victoria General Hospital, Seven Oaks General Hospital and Concordia Hospital) are community hospitals. All six hospitals submit patient discharge information to the WRHA. The WRHA maintains a Decision Support System (DSS), which includes hospital discharge abstracts containing patient identification, dates of admission and discharge, admitting attending physician, up to 25 diagnoses (coded using ICD, 10th Revision codes, with Canadian Enhancements [ICD10-CA]) - one for their primary [ie, most responsible] diagnosis and up to 24 additional ICD-10-CA codes) and 20 procedures (coded with Canadian Classification of Interventions codes) performed during the hospitalization. This DSS is used to aid in the planning of the services to the city.

\section{Sample selection}

Patients were identified for the present study from those admitted to any of the six hospitals in Winnipeg for at least one overnight stay between April 1, 2007 and March 31, 2012. All elderly (age $>65$ years of age) patients discharged with a primary diagnosis of IBD (identified from the DSS using ICD-10-CA codes K51 [UC] and K50 [CD]) were included. An approximately $20 \%$ random sample of each of the following groups were also included: elderly patients with secondary diagnosis of IBD, young (between 19 and 50 years of age) patients with a primary discharge diagnosis of IBD, and elderly patients with a primary discharge diagnosis of other colonic conditions (K52.3 [indeterminate colitis], K52.9 [noninfective gastroenteritis and colitis, unspecified], K55 [vascular disorders of intestine], K57.3 [diverticular disease of colon NOS], A04.7 [enterocolitis due to Clostridium difficile] or A09.9 [gastroenteritis and colitis of unspecified origin]).

Medical records were reviewed and the diagnosis of IBD was determined from review of the index hospitalization, subsequent hospitalization(s), ambulatory care visits with gastroenterologists and/or primary care physicians (including by reviewing the physician clinic records maintained separately from hospital records for patients with unclear diagnosis from hospital records), endoscopy and pathology reports. Follow-up was reviewed to August 2013. For individuals with a definite diagnosis of IBD, information regarding duration of disease, symptoms at presentation, extent of disease, extraintestinal manifestations (arthritis, iritis/uveitis, primary sclerosing cholangitis), IBD comorbidities (abdominal infections, fractures, infections associated with immunomodulator medications, venous thrombosis), hospital length of stay, use of IBD medications (5-aminosalicylates, azathioprine, 6-mercaptopurine, methotrexate, infliximab, adalimumab, cyclosporine, tacrolimus) before and during hospitalization, surgery (including type of surgery before and during hospitalization) and complications during hospitalization was extracted. The extent of disease was ascertained from the admission notes, endoscopy, pathology and/or medical imaging report(s). Medication usage before hospitalization was defined as any record of the patient ever being on the respective medication, including failed medical therapy and past hospitalizations.

\section{Analysis}

The accuracy of hospital discharge diagnosis codes for IBD in the elderly was assessed using the hospitalizations for the elderly with and without IBD diagnosis; each hospital admission was considered separately in the present analysis. For comparison of clinical features and management of IBD between the young and elderly with IBD, the first hospital admission during the study time period for those identified with the primary discharge diagnosis of IBD and documented IBD exacerbation on chart review were included. Individuals with mechanical bowel obstructions as determined from the discharge abstracts, medical imaging, endoscopy results and treatment course (conservative management versus treatment with IBD medications) were excluded.

Standard descriptive analysis was undertaken. Categorical data were compared (characteristics of younger and older individuals admitted with IBD) using Fisher's exact test. Logistic regression analyses were performed to assess potential predictors of use of an immunomodulator and/or biological agent in hospital (and before hospitalization in separate models) including age, sex, extent of disease, duration of disease, CD versus UC, comorbidities categorized using the Charlson comorbidity index (CCI), socioeconomic status (SES), admission at tertiary care versus community hospitals, and presence or absence of obstructive symptoms. The SES was determined using a previously validated index (Socioeconomic Factor Index), which is based on the characteristics of the neighbourhood of residence determined from Statistics Canada census data (5). A higher Socioeconomic Factor Index score indicates a more adverse SES. Parametric and nonparametric tests, as appropriate, were used for continuous data. The sensitivity, specificity, positive predictive value (PPV) and negative predictive value (NPV) of the IBD codes (K51 and K50) in the elderly patients were determined. Pearson correlation coefficient was used to assess correlation between primary hospital discharge abstract diagnosis (DAD) of IBD with IBD exacerbation and/or related obstruction on record review among the elderly.

\section{RESULTS}

Of the 395 admissions included in the present study, 82 were for individuals 19 to 50 years of age (all with primary discharge diagnosis of IBD) and 313 were for patients $>65$ years of age. Of the 313 admissions for patients $>65$ years of age, 178 were for a diagnosis of IBD whereas 135 were in the non-IBD group. In total, the study included 225 patients with IBD (158 CD; 67 UC) who experienced 260 admissions. While all admissions (primary or secondary diagnosis in the DAD) among the elderly were used to assess for accuracy of IBD codes in the DAD (because those are used in administrative database studies), for comparison of clinical characteristics among the elderly and young IBD patients, 138 patients with IBD as the primary discharge diagnosis who were admitted for an IBD exacerbation as determined from the chart reviews were included.

\section{Patient demographics and clinical presentation}

The elderly IBD patients were more likely to present with decreased oral intake, while the younger IBD patients were more likely to have inflammatory arthritis as an extraintestinal manifestation of their IBD (Table 1). There was a trend toward family history of IBD being more common in the younger population. There was no significant difference in the duration of IBD before admission. None of the patients admitted with an IBD exacerbation (or intestinal obstruction) died during their hospitalization.

\section{Clinical phenotype}

The extent of disease could be determined for $123 \mathrm{CD}$ patients (Table 2) and $53 \mathrm{UC}$ patients (Table 3). Of those with CD, younger patients were significantly more likely to have ileocolonic CD, whereas the elderly patients had disease restricted to either their colon or ileum. Of the younger CD patients, $16 \%$ had perianal disease compared with $3 \%$ of 
TABLE 1

Patient demographics and characteristics of individuals admitted with an inflammatory bowel disease (IBD) exacerbation and IBD primary discharge diagnosis

\begin{tabular}{|c|c|c|c|}
\hline \multirow[b]{2}{*}{ Characteristic } & \multicolumn{2}{|c|}{ Age, years } & \multirow[b]{2}{*}{$\mathbf{P}$} \\
\hline & $\begin{array}{c}>65 \\
(n=62)\end{array}$ & $\begin{array}{l}19-50 \\
(n=76)\end{array}$ & \\
\hline Male sex, $\%$ & 37 & 45 & 0.39 \\
\hline Age, years, median (IQR) & $73(69-78)$ & $31(25-41)$ & \\
\hline Crohn disease subjects, $\%$ & 63 & 64 & 0.86 \\
\hline $\begin{array}{l}\text { Duration of IBD before admission, } \\
\text { years, median }\end{array}$ & 6 & 7 & 0.17 \\
\hline IQR & $1-29$ & $0-14$ & \\
\hline Range & $0-59$ & $0-30$ & \\
\hline Hospital stay, nights, median (IQR) & $6.5(3-11)$ & $6(4-9)$ & 0.58 \\
\hline \multicolumn{4}{|l|}{ Symptoms at presentation, $\%$} \\
\hline Abdominal pain & 69 & 74 & 0.70 \\
\hline Decreased oral intake & 45 & 26 & 0.03 \\
\hline Diarrhea & 68 & 66 & 0.86 \\
\hline Failure to thrive & 27 & 28 & 1 \\
\hline Rectal bleeding & 39 & 47 & 0.39 \\
\hline Vomiting & 31 & 29 & 0.85 \\
\hline Family history of IBD, \% & 15 & 25 & 0.14 \\
\hline \multicolumn{4}{|l|}{ Extraintestinal manifestations, \% } \\
\hline Arthritis & 0 & 8 & 0.033 \\
\hline Iritis or uveitis & 0 & 3 & 0.50 \\
\hline Sclerosing cholangitis & 0 & 3 & 0.50 \\
\hline \multicolumn{4}{|l|}{ IBD complications, $\%$} \\
\hline Abdominal infections & 2 & 0 & 0.45 \\
\hline $\begin{array}{l}\text { Infections due to immunosuppressant } \\
\text { medications }\end{array}$ & 2 & 0 & 0.45 \\
\hline Fractures & 0 & 0 & \\
\hline Venous thrombosis & 5 & 1 & 0.33 \\
\hline \multicolumn{4}{|l|}{ Charlson comorbidity index score, \% } \\
\hline 0 & 73 & 96 & $<0.001$ \\
\hline 1 & 11 & 3 & \\
\hline 2 & 5 & 0.0 & \\
\hline$\geq 3$ & 11 & 1 & \\
\hline \multicolumn{4}{|l|}{ Discharge disposition, \% } \\
\hline Home & 98.4 & 98.7 & 1 \\
\hline Nursing home & 1.6 & 0 & \\
\hline Other hospital & 0 & 1.3 & \\
\hline Death & 0 & 0 & \\
\hline
\end{tabular}

IQR Interquartile range

the $>65$ years age group $(P<0.05)$. Of the elderly patients with IBD listed as primary discharge diagnosis, $16 \%$ presented with an obstruction comapred with $7 \%$ of younger patients $(\mathrm{P}=0.10)$. Extent of disease was not specified in the charts for $35 \mathrm{CD}$ patients (28 elderly, seven nonelderly) and in 14 UC patients (10 elderly, four nonelderly).

Medication use in IBD patients

The elderly IBD patients were more likely to be prescribed 5 -aminosalicylates in hospital or before admission, while the younger IBD patients were more likely to be prescribed biological therapy during hospitalization or before hospitalization (Table 4). Immunomodulators (azathioprine, 6-mercaptopurine, methotrexate) were commonly used in the younger population both before and during hospitalization. Corticosteroid use while in hospital was almost identical, with $77 \%$ of the older and $74 \%$ of the younger patients receiving them. Immunosuppressants (tacrolimus, cyclosporine) also showed similar usage between the two age groups.
TABLE 2

Extent of disease for Crohn disease patients admitted to hospital

\begin{tabular}{lcccc}
\hline & \multicolumn{2}{c}{ Age, years } & & \\
\cline { 2 - 3 } Disease extent & $\mathbf{7 5}$ & $\mathbf{1 9 - 5 0}$ & Total, $\mathbf{n}$ & $\mathbf{P}$ \\
\hline Colonic & $31(41.3)$ & $10(20.8)$ & 41 & 0.004 \\
Ileocolonic & $22(29.3)$ & $28(58.3)$ & 50 & \\
lleum only & $22(29.3)$ & $10(20.8)$ & 32 & \\
Total & 75 & 48 & 123 & \\
\hline
\end{tabular}

Data presented as $n$ (\%) unless otherwise indicated

TABLE 3

Extent of disease for ulcerative colitis patients admitted to hospital

\begin{tabular}{lcccc}
\hline & \multicolumn{2}{c}{ Age, years } & & \\
\cline { 2 - 3 } Disease extent & $\mathbf{7 6 5}$ & $\mathbf{1 9 - 5 0}$ & Total, $\mathbf{n}$ & $\mathbf{P}$ \\
\hline Proctosigmoiditis $^{*}$ & $6(20)$ & $2(8.7)$ & 8 & 0.24 \\
Left-sided colitis $^{\dagger}$ & $4(13.3)$ & $7(30.4)$ & 11 & \\
Extensive colitis $^{\ddagger}$ & $20(66.7)$ & $14(60.9)$ & 34 & \\
Total & 30 & 23 & 53 & \\
\hline
\end{tabular}

Data presented as $n$ (\%) unless otherwise indicated. *Rectum and/or sigmoid colon only $(<30 \mathrm{~cm})$; ${ }^{\dagger}$ Extending to involve descending colon and/or splenic flexure; ${ }^{\ddagger}$ Extending to involve transverse colon, hepatic flexure, ascending colon and/or cecum

Comorbidity and IBD

The CCI score and comorbidity burden was calculated from ICD$10-\mathrm{CA}$ codes in the DAD at the index admission. The older cohort was more likely to suffer from chronic pulmonary problems $(6.5 \%$ in $>65$ years versus $0 \%$ in $<50$ years; $\mathrm{P}<0.05)$, diabetes with complications $(8.1 \%$ in $>65$ years versus $0 \%$ in $<50$ years; $\mathrm{P}<0.05)$ and have a higher CCI score (Table 1).

Predictors of use of biological and/or immunomodulatory medications

In univariate analyses, the significant predictors of use of biological and/or immunomodulatory medications during the hospitalization were use of these medications before admission and younger IBD patient group. After adjusting for CCI score, these predictors continued to be significant predictors (Table 5). In analysis limited to the elderly with IBD, only previous use of these medications was a significant predictor of use of these medications during hospitalization (Table 5). Use of biologicals was limited to those with a CCI score of 0 and with young versus elderly IBD as the only significant predictor (OR 5.35 [95\% CI 1.1.3 to 25.24]). The only predictor of use of biologicals and/or immunomodulatory medications before hospitalization was age (young versus elderly IBD: OR 2.47 [95\% CI 1.21 to 5.05], adjusted for CCI score)

\section{Rates of surgery}

During the hospital stay, the younger IBD patients were more likely to undergo surgery $(37 \%)$ compared with the elderly population $(18 \%)$ $(\mathrm{P}<0.01)$. There was no difference in the rates of IBD surgery before admission between the two age groups (34\% of elderly versus $33 \%$ of younger IBD patients $[\mathrm{P}=1])$.

Accuracy of IBD ICD-10-CA codes

The ICD-10-CA codes for IBD in the elderly had an overall sensitivity of $100 \%$, a specificity of $81 \%$, a PPV of $87 \%$ and an NPV of $100 \%$. The sensitivity and specificity of ICD-10 codes were $98 \%$ and $96 \%$, respectively, in CD patients. The PPV was $94 \%$ and the NPV was $99 \%$ in elderly CD patients. The sensitivity and specificity were $98 \%$ and $92 \%$ in UC patients, respectively. The PPV in UC patients was $70 \%$ while the NPV was $99.6 \%$. There was no single predominant diagnosis among the miscoded cases. 
TABLE 4

Medication use in hospital and before admission in patients admitted with an inflammatory bowel disease (IBD) exacerbation and IBD primary discharge diagnosis

\begin{tabular}{|c|c|c|c|c|c|c|}
\hline \multirow[b]{3}{*}{ Medication } & \multicolumn{3}{|c|}{ Medication use in hospital } & \multicolumn{3}{|c|}{ Medication use before admission } \\
\hline & \multicolumn{2}{|c|}{ Age, years } & \multirow[b]{2}{*}{$\mathbf{P}$} & \multicolumn{2}{|c|}{ Age, years } & \multirow[b]{2}{*}{$\mathbf{P}$} \\
\hline & $>65(n=62)$ & $19-50(n=76)$ & & $>65(n=62)$ & $19-50(n=76)$ & \\
\hline Budesonide & 2 & 1 & 1 & 3 & 4 & 1 \\
\hline Biologicals* & 3 & 13 & 0.06 & 6 & 21 & 0.02 \\
\hline Mesalamine & 53 & 29 & 0.005 & 61 & 43 & 0.04 \\
\hline Immunomodulators $^{\dagger}$ & 13 & 24 & 0.13 & 27 & 43 & 0.07 \\
\hline Biologicals and/or immunomodulators & 16 & 33 & 0.03 & 29 & 47 & 0.03 \\
\hline
\end{tabular}

Data presented as \% unless otherwise indicated. *Includes adalimumab and infliximab; 'Includes azathioprine, 6-mercaptopurine and methotrexate; $¥$ Includes cyclosporine and tacrolimus

\section{TABLE 5}

Predictors of use of biological and/or immunomodulatory medications during hospitalization

\begin{tabular}{lcc}
\hline & \multicolumn{2}{c}{ OR $(95 \% \mathrm{Cl})$} \\
\cline { 2 - 3 } & \multicolumn{1}{c}{ Univariate } & Adjusted for $\mathbf{C C I}$ \\
\hline All inflammatory bowel disease patients $(\mathrm{n}=161)$ & \\
Young vs elderly & $3.21(1.46-7.02)$ & $2.82(1.23-6.44)$ \\
Use before hospitalization vs not & $9.30(4.05-21.35)$ & $9.07(3.93-20.93)$ \\
Elderly inflammatory bowel disease patients $(\mathrm{n}=79)$ & \\
Use before hospitalization vs not & 23.32 & 24.85 \\
& $(4.42-122.94)$ & $(4.59-134.60)$ \\
\hline
\end{tabular}

CCI Charlson comorbidity index; vs Versus

Use of primary discharge diagnosis of IBD in hospital records to detect admissions with IBD exacerbation and/or related intestinal obstruction

Ninety percent of elderly patients coded with IBD as the primary discharge diagnosis $(\mathrm{n}=79)$ were admitted for an IBD exacerbation and/or related obstruction, with a correlation of 0.70 between the two variables. Sixty-five percent (52 of 79) of elderly patients were admitted for an exacerbation without an obstruction, $13 \%$ for an exacerbation with an obstruction and $11 \%$ for an obstruction alone. Five UC and three $\mathrm{CD}$ admissions were coded with IBD as the primary discharge diagnosis, but the patient was not admitted due to an IBD exacerbation or bowel obstruction (Table 6). Of the 13 elderly patients with an IBD exacerbation and/or obstruction who were not coded with IBD as the primary discharge diagnosis, 12 were coded with a bowel obstruction (four mechanical) and one with an anal abscess as the primary discharge diagnoses. All 13 had CD and were not included in the comparison of clinical characteristics of young and elderly hospitalized with IBD because records of young patients with IBD as a secondary diagnosis in DAD were reviewed. Of note, the primary discharge diagnosis of IBD plus obstruction coding (among those with IBD as secondary code) identified all elderly patients with IBD admitted with IBD exacerbation and/ or intestinal obstruction. All but one case with an IBD exacerbation (anal abscess) was coded with IBD as the primary discharge diagnosis.

\section{DISCUSSION}

The present study suggests that hospitalized elderly patients with IBD are less likely to receive immunomodulators and/or biologicals during the hospitalization or before admission compared with younger IBD patients. While there may be a lower threshold to admit the elderly than younger persons with IBD, we were not able to identify an obvious difference in disease severity between elderly and young patients hospitalized with IBD, as indicated by comparable rates of surgery before admission, and corticosteroid use before and during hospitalization. Hence, the difference in utilization of immunomodulators and/or biologicals during hospitalizations may not be due to the

\section{TABLE 6}

Correlation of discharge abstract inflammatory bowel disease (IBD) primary diagnosis with IBD exacerbation/ bowel obstruction on chart review in the $>65$ years of age group

\begin{tabular}{lccc}
\hline \multirow{2}{*}{ Diagnosis } & \multicolumn{3}{c}{ Admission exacerbation/obstruction } \\
\cline { 2 - 4 } IBD secondary diagnosis & No & Yes & Total, $\mathbf{n}$ \\
IBD primary diagnosis & $51(79.7)$ & $13(20.3)$ & 64 \\
Total, $\mathrm{n}$ & $8(10.1)$ & $71(89.9)$ & 79 \\
\hline Data presented as & 59 & 84 & 143 \\
\hline
\end{tabular}

differences in severity of IBD in elderly versus younger individuals. Furthermore, the use of a single ICD-10-CA IBD code in the hospitalization discharge abstracts is valid and accurate in identifying elderly (>65 years of age) patients with IBD, although more so for CD than UC. The primary discharge diagnosis IBD coding identifies most elderly IBD patients admitted with IBD exacerbation. Cases with IBD exacerbation and/or obstruction can be identified by including those with IBD listed as primary discharge diagnosis; and those with IBD listed as a secondary diagnosis, concomitantly with obstruction as the primary discharge diagnosis.

Although there are limited data comparing hospitalized elderly and young IBD patients, the results of our comparisons of clinical characteristics of hospitalized IBD patients between the young and the elderly are consistent with the differences found in all (hospitalized and nonhospitalized) IBD patients in other studies. For example, previous studies, including those by Gower-Rousseau et al (1), Lakatos et al (2) and Quezada et al (6), have reported that elderly patients with IBD are less likely to have a family history of IBD. This trend may be due to older patients with IBD being less likely to have a strong genetic component to their disease compared with those diagnosed at a younger age. Environmental influence may play a larger role in those diagnosed with IBD at an older age. In our study, younger hospitalized $\mathrm{CD}$ patients were more likely to have ileocolonic disease compared with the older population, who was more likely to have their disease confined to the colon or terminal ileum, similar to that reported in all CD patients by Lakatos et al (1) and Quezada et al (6). Ileocolonic disease has been reported to be even more common in pediatric patients with IBD (7). However, we did not find a difference in the disease extent of UC in hospitalized elderly versus younger UC patients, in contrast to reports of more left-sided colitis in all (hospitalized and nonhospitalized) elderly UC patients (1). This may reflect similar rates of admission among elderly and younger UC patients with UC localized to different parts of the bowel.

We report a higher use of 5-aminosalicylates and lower use (both before admission and during hospitalization) of immunomodulators and/or biologicals among hospitalized elderly IBD patients. In contrast 
to previous studies, our study was limited to hospitalized patients and, hence, we believe this difference in utilization is not solely due to lower disease severity in the elderly because all patients included in the present study had disease sufficiently severe to warrant admission to hospital. The complications with immunomodulator and/or biological agent use have been reported to be more in the elderly, but complications of alternative forms of treatment, such as surgery and corticosteroids, are also higher in the elderly $(8,9)$. The response rate to the immunomodulator and/or biological agent use in the elderly has been reported to be similar to the younger individuals in observational studies $(8,10)$. Thus, cautious, but increased and earlier use of these more effective medications in the elderly should be considered.

Similarly, the lower rates of surgery among the hospitalized IBD elderly patients in our study may reflect clinician and/or patient preference to nonsurgical treatment. We recently reported that elderly IBD patients undergoing surgery had an increased risk for death compared with younger individuals (9). However, the prognosis of elderly UC patients undergoing emergency surgery is quite poor compared with those undergoing elective surgery (11). Thus, we recommend that surgical management in otherwise healthy elderly IBD patients should be promptly performed when necessary because increased morbidity and mortality can result from untreated or undertreated IBD exacerbations.

In our study, miscoded admissions were due to a wide variety of reasons including diverticulitis, colorectal cancer and surgical complications; however, there was not any particular gastroenterological condition for which ICD-10-CA miscoding was common. The specificity and PPV of the ICD-10-CA codes was higher in elderly CD patients than elderly UC patients, while the sensitivity and NPV were almost identical between the two groups. Thus, the single diagnostic ICD-10-CA code in discharge abstracts is sufficient to identify elderly CD patients, and a single code remains adequate to identify elderly UC patients. This finding is in contrast to studies investigating the use of ICD codes in records of ambulatory care visits. Hou et al (12) determined the accuracy of ICD-9 diagnostic codes for identifying IBD in the American Veterans Affairs health care system, using both inpatient and ambulatory care visits at two Veterans Affairs hospitals. They compared the ICD-9 diagnosis with the clinical diagnosis based on manual chart review and concluded that single ICD-9 codes are inadequate to define IBD patients. Similarly, Benchimol et al (13) concluded that the addition of a pharmacy claim was necessary to improve accuracy and supplement ICD codes,

\section{REFERENCES}

1. Lakatos P, David G, Pandur T. IBD in the elderly population: Results from a population-based study in Western Hungary, 1977-2008. J Crohns Colitis 2011;5:5-13.

2. Gower-Rousseau C, Vasseur F, Fumery M, et al. Epidemiology of inflammatory bowel diseases: New insights from a French populationbased registry (EPIMAD). Dig Liver Dis 2013;45:89-94.

3. Thia KT, Loftus E V, Sandborn WJ, et al. An update on the epidemiology of inflammatory bowel disease in Asia. Am J Gastroenterol 2008;103:3167-82.

4. Gisbert JP, Chaparro M. Systematic review with meta-analysis: Inflammatory bowel disease in the elderly. Aliment Pharmacol Ther 2014;39:459-77.

5. Chateau D, Metge C, Prior H, et al. Learning from the census: The socio-economic factor index (SEFI) and health outcomes in Manitoba. Can J Public Health 2012;103.

6. Quezada SM, Steinberger EK, Cross RK. Association of age at diagnosis and Crohn's disease phenotype. Age Ageing 2013;42:102-6.

7. Israeli E, Ryan JD, Shafer L-A, et al. Younger age at diagnosis is associated with panenteric, but not more aggressive, Crohn's disease. Clin Gastroenterol Hepatol 2014;12:72-9. when including ambulatory care visits. Overall, there has been very little research that has examined the accuracy of ICD codes in elderly IBD patients and/or only in the hospital discharge abstracts. Our study suggests that ICD-10 codes can be very accurate in identifying the elderly hospitalized IBD population when used by welltrained coders. Our sample population was selected by ICD-10 codes for IBD (K50 or K51) and other gastrointestinal disorders theoretically more likely to be miscoded as IBD. Hence, the PPV and specificity will likely be higher when assessing value of IBD ICD codes in the general population and not just limited to those with disorders, which are more likely to be miscoded as IBD.

The results of the current study should be interpreted in the context of its strengths and limitations. We evaluated a citywide practice and, hence, the results should be more applicable to usual clinical practice than the studies from specialized centres. We evaluated ICD codes in hospitalized patients and the results may not be applicable to outpatient physician claims datasets. However, other gastrointestinal conditions (eg, ischemic colitis), which are more common in the elderly and have a risk for miscoding as IBD in the discharge abstracts, are more likely to lead to hospitalization and, therefore, may be less of an issue in outpatient settings. Hence, administrative data coding accuracy among young and elderly IBD patients in outpatient settings is likely to be similar. We did not evaluate clinical presentation or management according to age of onset/diagnosis of IBD. We did not assess initiation of immunomodulators and/or biologicals immediately postdischarge from the hospitals, or clinical course after discharge from the hospitals.

\section{CONCLUSION}

Our study suggests that immunomodulators and biological agents and surgery are used less often in elderly hospitalized IBD patients. Additional studies should assess approaches to increase the use of immunomodulators and/or biological agents in elderly patients with IBD. Single ICD-10 CA codes can be used to identify elderly hospitalized individuals with IBD, in particular those admitted with IBD exacerbations.

DISCLOSURES: The authors have no financial disclosures or conflicts of interest to declare.

8. Ljung T. Infliximab in inflammatory bowel disease: Clinical outcome in a population based cohort from Stockholm County. Gut 2004;53:849-53.

9. Bernstein CN, Nugent Z, Targownik LE, Singh H, Lix LM. Predictors and risks for death in a population-based study of persons with IBD in Manitoba. Gut 2014 (Epub ahead of print).

10. Moleski SM, Lindenmeyer CC, Kozuch PL. The use of infliximab in older inflammatory bowel disease patients. Gastroenterology 2011;142:S-361.

11. Ikeuchi H, Uchino M, Matsuoka H, et al. Prognosis following emergency surgery for ulcerative colitis in elderly patients. Surg Today 2014;44:39-43.

12. Hou JK, Tan M, Stidham RW, et al. Accuracy of diagnostic codes for identifying patients with ulcerative colitis and Crohn's disease in the Veterans Affairs health care system. Dig Dis Sci 2014:7-11.

13. Benchimol EI, Guttmann A, Mack DR, et al. Validation of international algorithms to identify adults with inflammatory bowel disease in health administrative data from Ontario, Canada. J Clin Epidemiol 2014;67:887-96. 


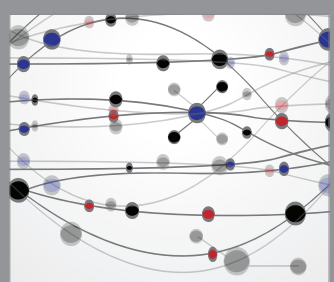

The Scientific World Journal
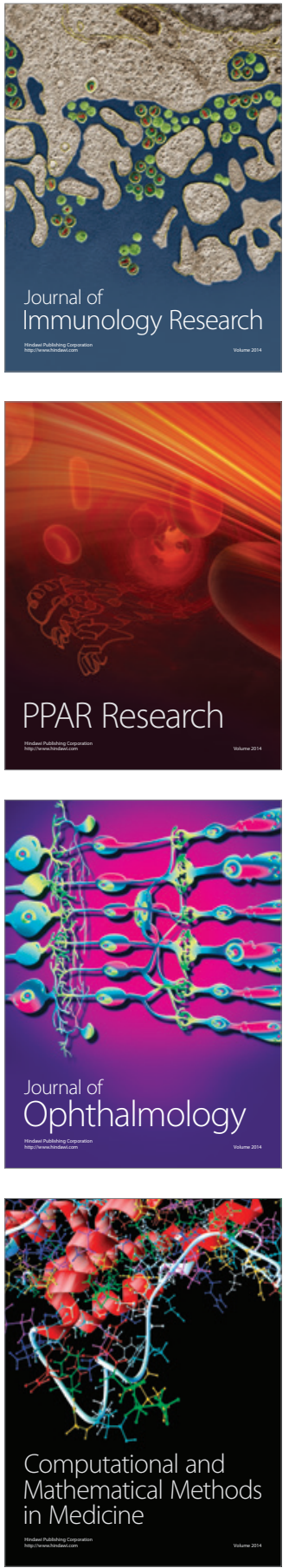

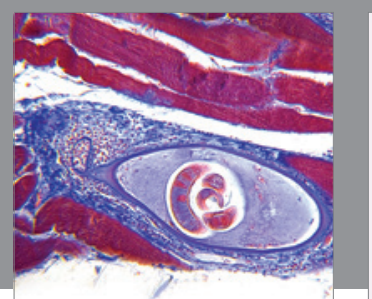

Gastroenterology Research and Practice

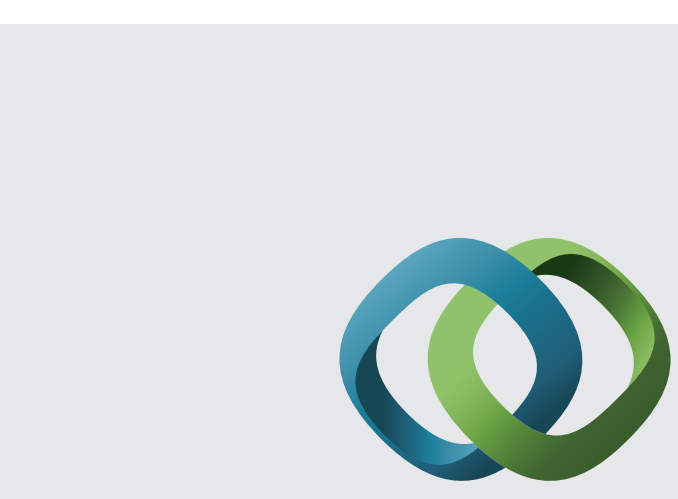

\section{Hindawi}

Submit your manuscripts at

http://www.hindawi.com
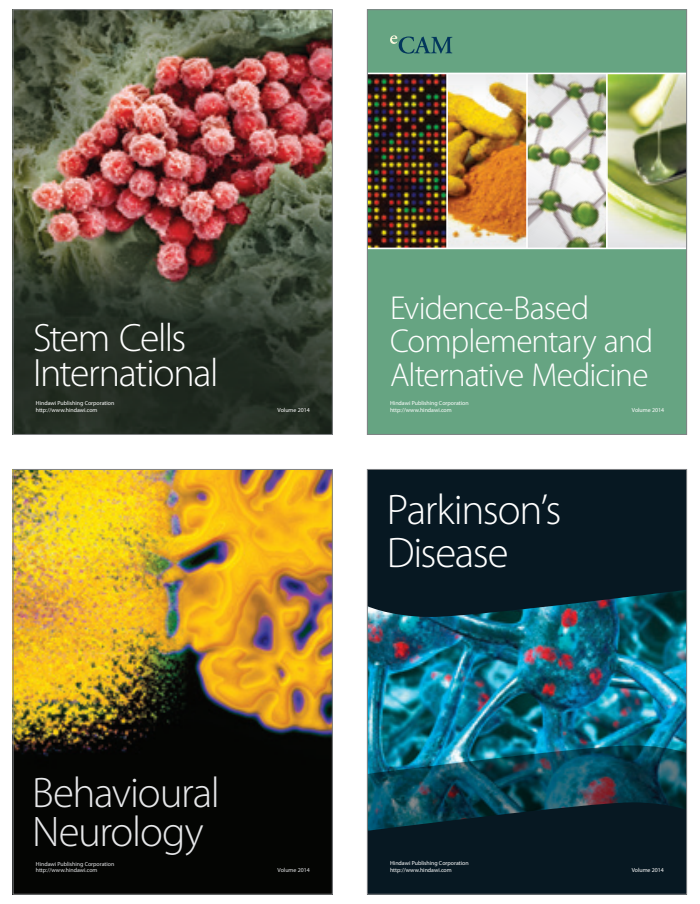
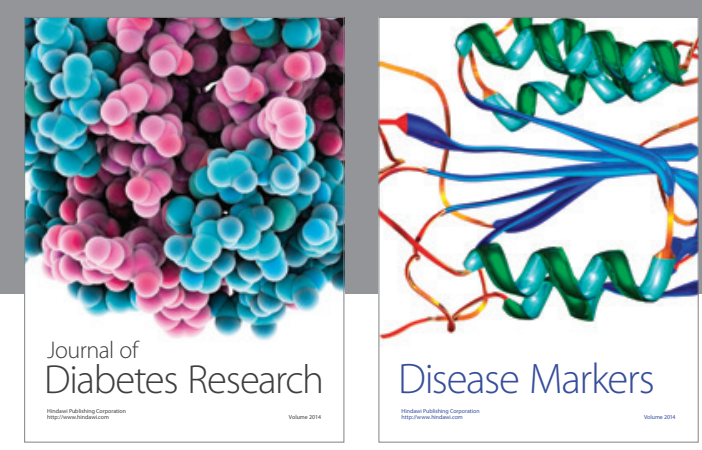

Disease Markers
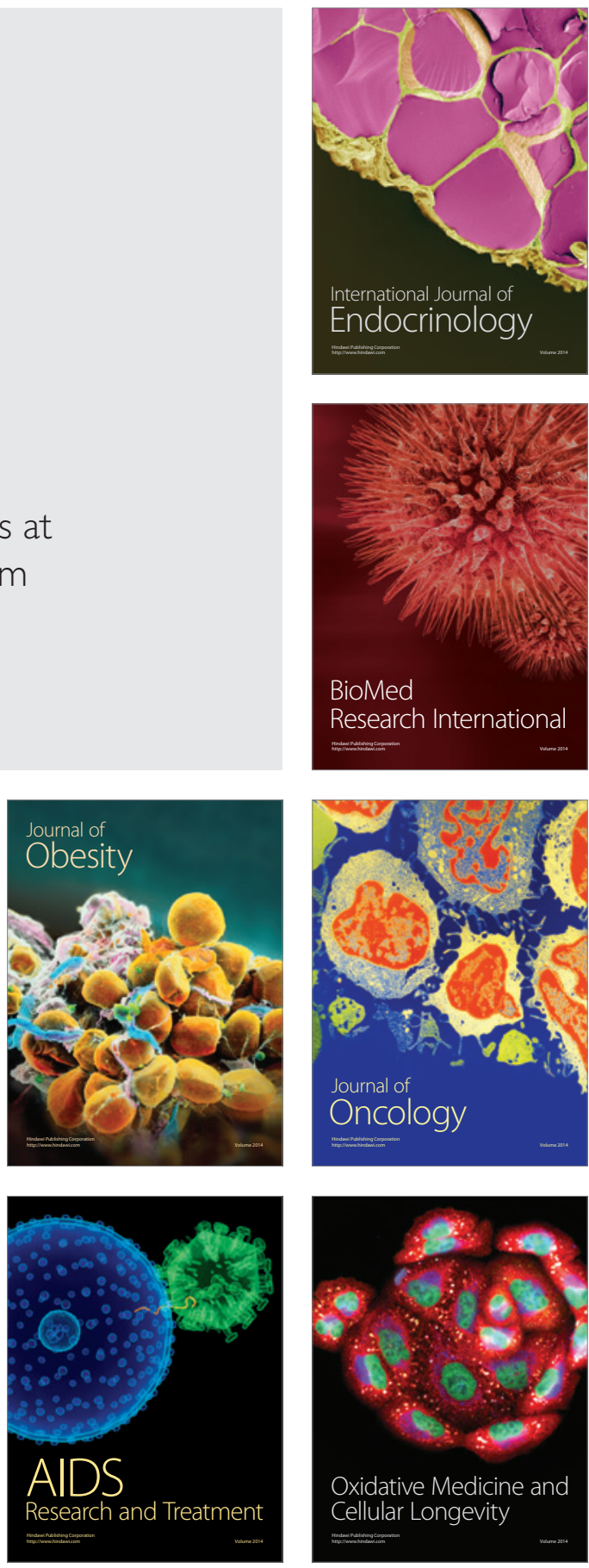